\title{
Twitter-Derived Social Neighborhood Characteristics and Individual-Level Cardiometabolic Outcomes: Cross-Sectional Study in a Nationally Representative Sample
}

Dina Huang ${ }^{1}$, PhD; Yuru Huang ${ }^{1}$, MHS; Sahil Khanna ${ }^{2}$, MSc; Pallavi Dwivedi ${ }^{1}$, MSc; Natalie Slopen ${ }^{1}$, ScD; Kerry M Green ${ }^{3}, \mathrm{PhD}$; Xin $\mathrm{He}^{1}, \mathrm{PhD}$; Robin Puett ${ }^{4}, \mathrm{PhD}$; Quynh Nguyen ${ }^{1}, \mathrm{PhD}$

\footnotetext{
${ }^{1}$ Department of Epidemiology and Biostatistics, University of Maryland School of Public Health, College Park, MD, United States

${ }^{2}$ A. James Clark School of Engineering, University of Maryland, College Park, MD, United States

${ }^{3}$ Department of Behavioral and Community Health, University of Maryland School of Public Health, College Park, MD, United States

${ }^{4}$ Maryland Institute for Applied Environmental Health, University of Maryland School of Public Health, College Park, MD, United States
}

\section{Corresponding Author:}

Dina Huang, $\mathrm{PhD}$

Department of Epidemiology and Biostatistics

University of Maryland School of Public Health

4200 Valley Drive

College Park, MD, 20742

United States

Phone: 16107879386

Email: dinahuang26@gmail.com

\section{Abstract}

Background: Social media platforms such as Twitter can serve as a potential data source for public health research to characterize the social neighborhood environment. Few studies have linked Twitter-derived characteristics to individual-level health outcomes.

Objective: This study aims to assess the association between Twitter-derived social neighborhood characteristics, including happiness, food, and physical activity mentions, with individual cardiometabolic outcomes using a nationally representative sample.

Methods: We collected a random 1\% of the geotagged tweets from April 2015 to March 2016 using Twitter's Streaming Application Interface (API). Twitter-derived zip code characteristics on happiness, food, and physical activity were merged to individual outcomes from restricted-use National Health and Nutrition Examination Survey (NHANES) with residential zip codes. Separate regression analyses were performed for each of the neighborhood characteristics using NHANES 2011-2016 and 2007-2016.

Results: Individuals living in the zip codes with the two highest tertiles of happy tweets reported BMI of 0.65 (95\% CI -1.10 to -0.20$)$ and $0.85 \mathrm{~kg} / \mathrm{m}^{2}$ (95\% CI -1.48 to -0.21$)$ lower than those living in zip codes with the lowest frequency of happy tweets. Happy tweets were also associated with a 6\%-8\% lower prevalence of hypertension. A higher prevalence of healthy food tweets was linked with an $11 \%$ (95\% CI $2 \%$ to $21 \%$ ) lower prevalence of obesity. Those living in areas with the highest and medium tertiles of physical activity tweets were associated with a lower prevalence of hypertension by $10 \%$ (95\% CI $4 \%$ to $15 \%$ ) and $8 \%$ ( $95 \%$ CI $2 \%$ to $14 \%$ ), respectively.

Conclusions: Twitter-derived social neighborhood characteristics were associated with individual-level obesity and hypertension in a nationally representative sample of US adults. Twitter data could be used for capturing neighborhood sociocultural influences on chronic conditions and may be used as a platform for chronic outcomes prevention.

(JMIR Public Health Surveill 2020;6(3):e17969) doi: $10.2196 / 17969$

\section{KEYWORDS}

neighborhood study; cardiometabolic outcomes; Twitter 


\section{Introduction}

The neighborhood environment has been recognized as an important determinant of health. Previous studies have identified associations between neighborhood characteristics and health behaviors [1,2], chronic conditions [3,4], and mental health outcomes $[5,6]$. Access to healthy food, proximity to parks, recreational facilities, and neighborhood walkability are protective factors of obesity, diabetes, and hypertension [7-10]. Conversely, neighborhood disadvantage and neighborhood-level stressors are associated with higher prevalence of obesity and hypertension [3,11-13].

In addition to the physical environment, social contextual factors are also associated with a variety of health outcomes. The Roseto Effect describes the phenomenon in which members of a close-knit community experience a lower heart rate than members of a neighborhood community and is an example of the potential influence of the social environment [14]. Research has shown that greater community happiness is associated with decreased prevalence of obesity, hypertension, and suicide, as well as increased life expectancy [15-17]. Greater social cohesion has been linked with lower hypertension [7,11], and social capital has been linked with lower prevalence of obesity, hypertension, and mental health conditions [18,19]. Social media, such as Twitter, can serve as a data source to characterize the social neighborhood environment. Previous studies using Twitter data have validated the approach for assessing dietary choices, measuring happiness, and examining community levels of physical activity [20-22]. Unlike traditional indicators of neighborhood environment, Twitter indicators reflect an individual's perception and attitude towards the neighborhood environment, as well as an individual's use of neighborhood resources [23]. Traditional neighborhood studies mainly rely on time-consuming neighborhood data collection within limited geographical areas. In comparison, Twitter-derived indicators as proxy measures for neighborhood factors provide low-cost opportunities to conduct neighborhood studies at the national level and to study the association between geographic factors and health outcomes. We hypothesize that neighborhood-level factors, as estimated by aggregating information from tweets, influence individual-level health.

\section{Underlying Mechanism}

According to social learning theory, learning occurs in a social context [24]. Social context influences individual health behaviors through reciprocal interactions between people, as well as between people and the environment, through observational learning of modeled behaviors, self-initiated reinforcement, or external positive or negative reinforcement, and socially communicated expectations of particular health behaviors [24]. For instance, communities that tweet more about physical activity may culturally support such activity more than other communities, thus reinforcing the decision of a given resident to engage in similar activity. Communities might also differ in the foods they prefer; therefore, utilizing Twitter data, we can estimate food preferences and norms and determine whether these relate to health outcomes on an individual level.

\section{Study Aim and Hypothesis}

In this study, we utilized Twitter-derived characteristics, including happiness, food, and physical activity, as social neighborhood predictors. We assessed the associations between the Twitter-derived characteristics and cardiometabolic outcomes, including obesity, diabetes, and hypertension, using a nationally representative sample from the National Health and Nutrition Examination Survey (NHANES). We hypothesized that people living in zip codes with high levels of Twitter-derived neighborhood happiness, healthy diet, and physical activity have lower mean BMI and lower prevalence of obesity, diabetes, and hypertension, respectively.

\section{Methods}

\section{Study Population and Outcomes}

Individual-level health data were obtained from the NHANES 2007-2008, 2009-2010, 2011-2012, 2013-2014, and 2015-2016. We received approval to access the restricted, geocoded data from the National Center for Health Statistics (NCHS) Restricted Data Center (RDC). NHANES data and Twitter-derived predictors were merged via zip code linkages by an NCHS analyst. Zip codes were masked after data linkage. All statistical analyses were performed at the Maryland Federal Statistical Research Data Center, and all output was reviewed by an RDC staff member to avoid information disclosure. We followed the RDC confidentiality and disclosure review policies to protect the confidentiality of the NCHS study participants.

NHANES data consist of interview data (demographic, socioeconomic, and health-related questions) and examination data (physiological checks as well as laboratory tests). Data collection for NHANES was approved by the NCHS Research Ethics Review Board (ERB). Analysis of deidentified data from the survey is exempt from the federal regulations for the protection of human research participants. Analysis of restricted data through the NCHS RDC is also approved by the NCHS ERB. The study was approved by the University of Maryland Institutional Review Board (IRB).

We examined the following cardiometabolic outcomes: BMI, obesity, diabetes, and hypertension. NHANES measured weight and height data were used to calculate BMI. Obesity was defined as $\mathrm{BMI} \geq 30 \mathrm{~kg} / \mathrm{m}^{2}$. BMI and obesity are interdependent outcomes. Given BMI is a continuous variable, it provides more statistical power to detect differences, enabling readers to assess how much Twitter-derived variables might shift the distribution of BMI values. However, obesity is a clinically important health outcome. We present analyses with both to provide a more comprehensive examination of associations between Twitter-derived community variables and health outcomes. Hypertension was defined as having elevated blood pressure or self-report of taking medications for hypertension. A mean systolic blood pressure $>130 \mathrm{~mm} \mathrm{Hg}$ or mean diastolic blood pressure $>80 \mathrm{~mm} \mathrm{Hg}$ was defined as elevated blood pressure [25]. Diabetes was defined as having a glycohemoglobin (\%) value $\geq 6.5 \%$ or self-reported diagnoses of diabetes [26].

We included both individual-level and zip code-level covariates to account for confounding. Individual-level covariates from 
NHANES included age, sex, race/ethnicity, and annual household income. Zip code level characteristics included the following: percent of non-Hispanic white, median household income, population density, and median age obtained from the 2011-2015 American Community Survey 5-year estimates [27]. To avoid disclosure of zip codes, we replaced continuous percent non-Hispanic white, median age, and population density with the corresponding median value in each 20-quantile group. We replaced continuous median household income with the corresponding log-transformed median value for each 20-quantile group as requested by the RDC.

Twitter-Derived Social Neighborhood Characteristics

A random $1 \%$ of the geotagged tweets that are publicly available were collected through Twitter's streaming application programming interface (API) from February 2015 to March 2016. Geotagged tweets have the latitude and longitude coordinates of the location from which they were sent. We collected 79,848,992 geotagged tweets across the contiguous United States (including Washington, DC) and identified 603,363 unique Twitter users. Duplicated tweets and tweets identified as job postings through hashtags were removed. Each tweet was linked to the corresponding zip code through spatial join using Python (Python Software Foundation) [28].

Detailed information on the construction and validation of Twitter characteristics can be found in a previously published article [20]. Figure 1 is a flowchart of Twitter data collection and the construction of Twitter characteristics. Here, we briefly summarized the process to construct Twitter characteristics. We implemented sentiment analysis with the Machine Learning for Language Toolkit (MALLET) [29] to compute the happiness score (range from 0 to 1 ) for each tweet. We included diverse sources of training data such as Sentiment140 [30], Sanders Analytics [31], and Kaggle [32]. A binary variable of happiness was created for each tweet based on the MALLET score, where a rating $>0.8$ was defined as happy. The cut-off point of 0.8 reached the highest accuracy of classifying happy tweets and maintained the same prevalence of happy tweets identified in the human-labeled dataset [20]. After identifying each tweet as "happy" versus "not happy," we calculated the percent of happy tweets within each zip code [33].

For food analysis, we created a list of over 1430 popular food words from the US Department of Agriculture's National Nutrient Database [34]. Fruits, vegetables, nuts, and lean proteins were labeled as healthy food $(n=340)$, and fast food labels were also used $(n=154)$. We identified 4,041,521 food-related tweets and filtered each tweet by items on the food list to categorize them as mentions of healthy or fast food. The percentages of healthy and fast food tweets were calculated at the zip code level.

Similar to our food analysis, we created a list of physical activities using the published list of physical activity terms collected from physical activity questionnaires, a compendium of physical activities, and popular fitness programs [35-37]. A total of 376 items were gathered and included activities from gym exercise, sports, recreational activities, and household chores. Expressions such as "running late" and "walk away" were excluded. To avoid including tweets about passively watching sports, we excluded tweets with the verbs "watch/watching/watches/watched" and "attend/attends/attending/attending" and only included team sports tweets when there was the word “play/plays/playing/played.” We collected 1,473,976 geotagged tweets associated with physical activity. The percentage of physical activity tweets was aggregated at the zip code level.

To comply with RDC confidentiality and disclosure review policies, we were unable to use continuous percent Twitter characteristics at the zip code level, since the data may serve as geo-identifiers. We categorized Twitter characteristics at the zip code level into tertiles (high, medium, low) as predictors. 
Figure 1. Twitter data collection and construction of Twitter characteristics.

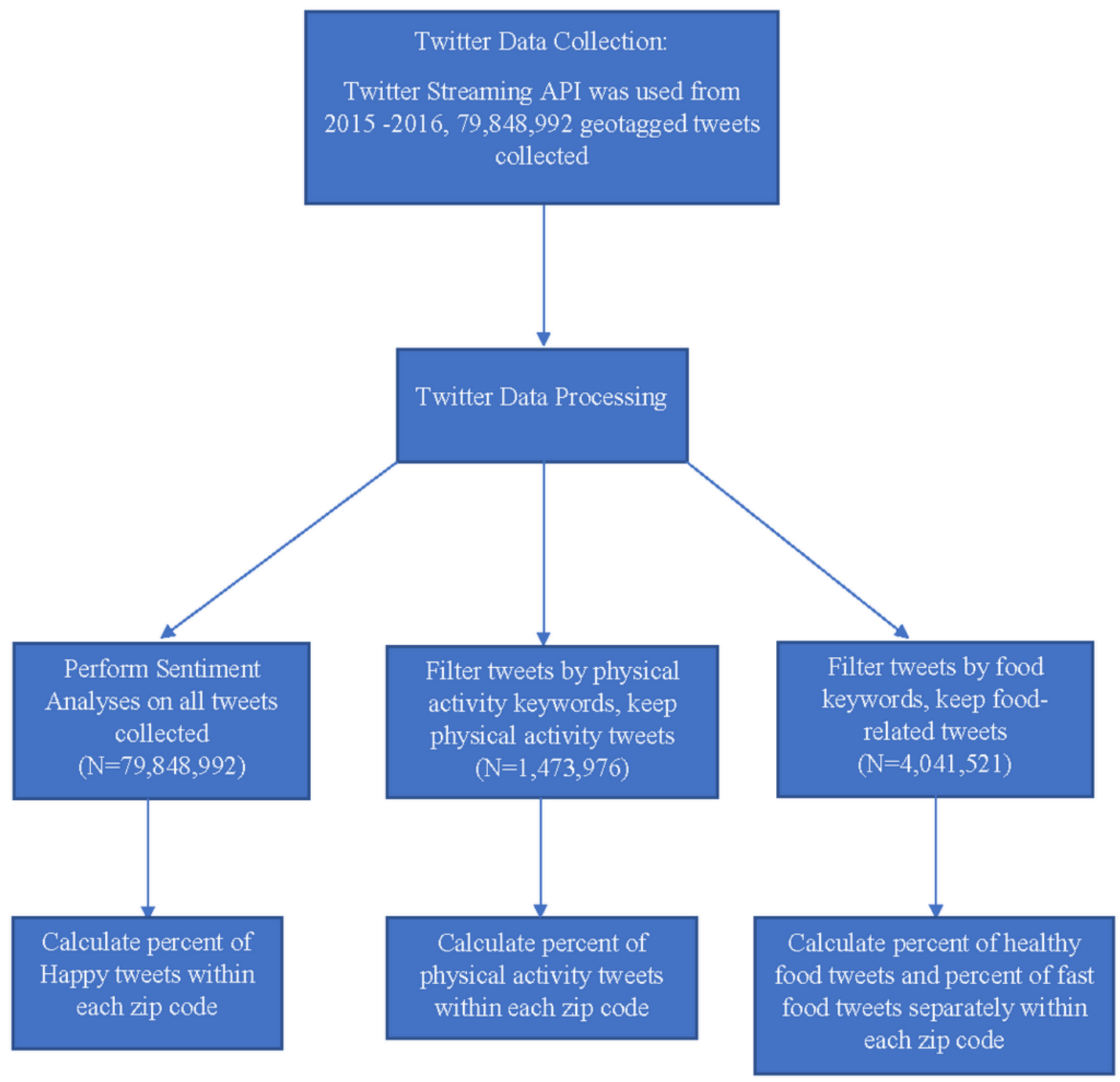

\section{Statistical Analysis}

We implemented separate regression analyses for each of the outcomes. Linear regression was used for continuous outcomes such as BMI; Poisson regression was used for binary outcomes including obesity, diabetes, and hypertension, to estimate prevalence ratios [38]. All models controlled for individual-level demographics and zip code-level characteristics. We analyzed health outcomes for the NHANES 2011-2016 subcohort, which is closer in time to the Twitter data (2015-2016). As a supplement, we analyzed NHANES data from the most recent five survey cycles from 2007 to 2016 (described below as the "full cohort") to obtain a sample with a higher diversity of zip codes (2116 zip codes in the full cohort and 1384 zip codes for the subcohort). A 10-year Mobile Examination Center (MEC) weight was used for NHANES data from 2007 to 2016, and a 6-year MEC weight was used for NHANES data from 2011 to $2016[39,40]$. Analyses were performed in Stata MP15 (StataCorp LP).

\section{Results}

\section{Descriptive Statistics}

Descriptive statistics are shown in Tables 1 and 2. The zip code-level Twitter characteristics were calculated for all zip codes in the United States. Of these, 19\% were happy $(n=29,606), 2.2 \%$ mentioned physical activity, and $5.0 \%$ mentioned food. There were fewer tweets about healthy foods $(1.0 \%)$ and fast food $(0.3 \%)$. Examples of each Twitter-derived characteristic are listed in Table 3.

For the full cohort, the mean age was 47 years, and 15,040 of 29,201 participants $(51.9 \%)$ were female. Reported participant race and ethnicity included $12,113(66.6 \%)$ non-Hispanic white, 7627 (14\%) Hispanic, 6179 (11\%) non-Hispanic black, and $3282(8 \%)$ identified as other races. The mean BMI was 29 $\mathrm{kg} / \mathrm{m}^{2}$, and the prevalence of obesity was $36.5 \%(10,478$ participants). The mean glycohemoglobin level was 5.6\%, and the prevalence of diabetes was $12.1 \%(n=4603)$. Hypertension was reported in 14,336 participants $(48.1 \%)$. Individual demographic characteristics in the subcohort were similar to those in the full cohort. 
Table 1. Descriptive characteristics for Twitter social neighborhood characteristics.

\begin{tabular}{lll}
\hline Zip code level Twitter characteristics & Number of zip codes with Twitter characteristics & Mean percentage (SE) \\
\hline Happy tweets & 29,606 & $19.0(0.06)$ \\
Tweets about physical activity & 29,604 & $2.2(0.02)$ \\
Tweets about food & 24,177 & $5.0(0.03)$ \\
Tweets about healthy food & 24,173 & $1.0(0.02)$ \\
Tweets about fast food & 24,174 & $0.3(0.01)$ \\
\hline
\end{tabular}

Table 2. Descriptive characteristics for individual characteristics from the National Health and Nutrition Examination Survey.

\begin{tabular}{|c|c|c|c|c|}
\hline \multirow[t]{2}{*}{ Individual-level characteristics } & \multicolumn{2}{|c|}{ NHANES $^{\mathrm{a}} 2007-2016^{\mathrm{b}}$} & \multicolumn{2}{|l|}{ NHANES 2011-2016 ${ }^{\mathrm{c}}$} \\
\hline & Total participants, $\mathrm{n}$ & Mean, \% (SE) & Total participants, $\mathrm{n}$ & Mean, $\%$ (SE) \\
\hline Age (years), mean (SE) & 29,201 & $47.3(0.25)$ & 17,048 & $47.6(0.36)$ \\
\hline Female, \% (SE) & 15,040 & $51.9(0.28)$ & 8803 & $52.0(0.40)$ \\
\hline Married, \% (SE) & 14,836 & $55.0(0.72)$ & 8534 & $54.2(1.02)$ \\
\hline \multicolumn{5}{|l|}{ Race/Ethnicity, \% (SE) } \\
\hline Black, non-Hispanic, \% (SE) & 6179 & $11.4(0.82)$ & 3830 & $11.4(1.17)$ \\
\hline White, non-Hispanic, \% (SE) & 12,113 & $66.6(1.62)$ & 6376 & $65.4(2.13)$ \\
\hline Hispanic, \% (SE) & 7627 & $14.3(1.11)$ & 4156 & $14.8(1.45)$ \\
\hline \multicolumn{5}{|l|}{ Education, \% (SE) } \\
\hline Less than high school & 7579 & $17.2(0.70)$ & 3942 & $15.5(0.97)$ \\
\hline High school & 6596 & $22.1(0.55)$ & 3708 & $20.9(0.71)$ \\
\hline Some college & 8366 & $31.4(0.52)$ & 5119 & $32.5(0.76)$ \\
\hline College or greater & 6621 & $29.3(1.02)$ & 4262 & $31.2(1.45)$ \\
\hline \multicolumn{5}{|c|}{ Total annual household income (US\$), \% (SE) } \\
\hline$<20,000$ & 6247 & $15.0(0.61)$ & 3593 & $14.9(0.85)$ \\
\hline $20,000-55,000$ & 11,518 & $37.1(0.67)$ & 6453 & $36.1(0.97)$ \\
\hline $55,000-75,000$ & 2965 & $12.6(0.44)$ & 1709 & $12.3(0.59)$ \\
\hline $75,000-100,000$ & 2503 & $11.4(0.38)$ & 1437 & $10.8(0.41)$ \\
\hline$\geq 100,000$ & 4399 & $23.9(1.09)$ & 2861 & $25.9(1.61)$ \\
\hline BMI $\left(\mathrm{kg} / \mathrm{m}^{2}\right)$, mean $(\mathrm{SE})$ & 28,818 & $28.9(0.09)$ & 16,830 & $29.1(0.12)$ \\
\hline Obesity, \% (SE) & 10,478 & $36.5(0.54)$ & 6144 & $37.6(0.76)$ \\
\hline Hemoglobin $\mathrm{A}_{1 \mathrm{c}}, \%$ (SE) & 27,775 & $5.6(0.01)$ & 16,280 & $5.6(0.01)$ \\
\hline Diabetes prevalence, $\%(\mathrm{SE})$ & 4603 & $12.1(0.32)$ & 2741 & $12.6(0.42)$ \\
\hline Hypertension, \% (SE) & 14,336 & $48.1(0.59)$ & 8411 & $48.8(0.77)$ \\
\hline
\end{tabular}

${ }^{\mathrm{a}}$ NHANES: National Health and Nutrition Examination Survey.

${ }^{\mathrm{b}}$ Descriptive statistics were weighted using the Mobile Examination Center 10-year weight.

${ }^{\mathrm{c}}$ Descriptive statistics were weighted using Mobile Examination Center 6-year weight. 
Table 3. Examples of each Twitter characteristic ${ }^{\mathrm{a}}$.

\begin{tabular}{|c|c|c|c|c|}
\hline Example number & Happy tweets & Fast food tweets & Healthy food tweets & Physical activity \\
\hline Example 1 & $\begin{array}{l}\text { "I am so blessed that my family is } \\
\text { healthy - it is all it matters!" }\end{array}$ & $\begin{array}{l}\text { "I just left pizzahut with } \\
\text { my mother!" }\end{array}$ & "collard greens are so delicious" & $\begin{array}{l}\text { "gotta get up and workout in } \\
\text { a couple hours hopefully I can } \\
\text { get up } \mathscr{\circ} \text { ", }\end{array}$ \\
\hline Example 2 & $\begin{array}{l}\text { "Me \& my bestie celebrating her } \\
\text { bachelorette trip. We are having a } \\
\text { blast!" }\end{array}$ & $\begin{array}{l}\text { "The perfect afternoon } \\
\text { work spot @starbucks" }\end{array}$ & $\begin{array}{l}\text { "Today woke up at } 8 \text { am to eat a } \\
\text { kale salad" }\end{array}$ & $\begin{array}{l}\text { "I just finished running } 6.02 \\
\text { miles in } 50 \mathrm{~m}: 44 \mathrm{~s} "\end{array}$ \\
\hline Example 3 & $\begin{array}{l}\text { "Wednesday night with the best } \\
\text { people!" }\end{array}$ & "Taco Bell run" & $\begin{array}{l}\text { "I cooked for lunch today! Brown } \\
\text { rice with roast beef, broccoli, and } \\
\text { green beans - yummm!" }\end{array}$ & $\begin{array}{l}\text { "A fun seven-mile hike at } \\
\text { Shenandoah" }\end{array}$ \\
\hline Example 4 & $\begin{array}{l}\text { "Brunch after the hike!!!\#food- } \\
\text { porn" }\end{array}$ & $\begin{array}{l}\text { "Chipotle line mad long } \\
\text { but I am not leaving!" }\end{array}$ & $\begin{array}{l}\text { "Turkey, broccoli, spinach, and } \\
\text { tomatoes! This is breakfast yay" }\end{array}$ & $\begin{array}{l}\text { "hiked to the summit of a } \\
\text { mountain today!" }\end{array}$ \\
\hline
\end{tabular}

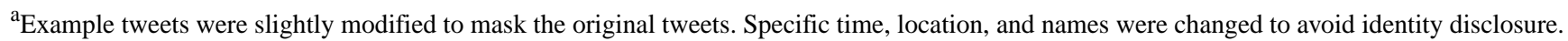

\section{Regression Results}

Zip code-level happiness was associated with lower mean BMI, as well as a lower prevalence of hypertension (Table 4). Comparing individuals living in the medium (second tertile) and the highest (third tertile) to the lowest level (first tertile) of happy tweets, mean BMI decreased by $0.65 \mathrm{~kg} / \mathrm{m}^{2}(95 \% \mathrm{CI}$ -1.10 to -0.20$)$ and $0.85 \mathrm{~kg} / \mathrm{m}^{2}(95 \% \mathrm{CI}-1.48$ to -0.21$)$, respectively. The prevalence of hypertension was lower by $8 \%$ (prevalence ratio [PR] $0.92 ; 95 \%$ CI 0.86 to 0.98 ) and $6 \%$ (PR $0.94 ; 95 \%$ CI 0.88 to 1.00 ) in the medium and highest tertiles versus the lowest tertile (Table 4). Associations between happy tweets and obesity and diabetes bordered on statistical significance in the subcohort analyses, but were statistically significant in the full cohort analyses.

High levels of Twitter-derived physical activity were associated with a lower prevalence of hypertension. In a comparison of individuals living in zip codes in the medium and highest levels of physical activity tweets to those with the lowest level, hypertension decreased by $8 \%$ (PR $0.92,95 \%$ CI 0.87 to 0.98 ) and $10 \%$ (PR 0.90, 95\% CI 0.85 to 0.96 ), respectively. Physical activity tweets were not associated with BMI, obesity, and diabetes.

Healthy food tweets were linked to BMI, obesity, and hypertension. Individuals living in zip codes with medium and high levels of healthy food tweets had mean BMI values that were $0.73 \mathrm{~kg} / \mathrm{m}^{2}$ lower (95\% CI -1.39 to -0.07$)$ and $1.02 \mathrm{~kg} / \mathrm{m}^{2}$ lower (95\% CI -1.39 to -0.07$)$. The prevalence of obesity was $5 \%$ (PR $0.95,95 \%$ CI 0.86 to 1.04 ) and $11 \%$ lower (PR 0.88, $95 \%$ CI 0.79 to 0.98 ) and the prevalence of hypertension was $6 \%$ (PR $0.94,95 \%$ CI 0.88 to 1.00 ) and $1 \%$ (PR $0.99,95 \%$ CI 0.91 to 1.06 ) lower. Fast food tweets were not associated with BMI, obesity, hypertension, and diabetes. Table 4 shows the number of study participants with given characteristics.

In supplemental analyses using NHANES 2007-2016 (Table 5), we observed associations that exhibited similar patterns as the regression results using the subcohort, with some stronger associations. Table 5 shows the number of study participants with given characteristics. 
Table 4. Twitter-derived neighborhood characteristics and adult health outcomes in the NHANES 2011-2016 subcohort ${ }^{\mathrm{a}}$.

\begin{tabular}{|c|c|c|c|c|}
\hline $\begin{array}{l}\text { Zip code-level Twitter predic- } \\
\text { tors and tertiles }\end{array}$ & $\operatorname{BMI}\left(\mathrm{kg} / \mathrm{m}^{2}\right), \mathrm{b}(95 \% \mathrm{CI})^{\mathrm{b}}$ & $\begin{array}{l}\text { Obesity, prevalence ratio } \\
(95 \% \mathrm{CI})^{\mathrm{b}}\end{array}$ & $\begin{array}{l}\text { Hypertension, prevalence ratio } \\
(95 \% \mathrm{CI})^{\mathrm{b}}\end{array}$ & $\begin{array}{l}\text { Diabetes, prevalence ratio } \\
(95 \% \mathrm{CI})^{\mathrm{b}}\end{array}$ \\
\hline \multicolumn{5}{|l|}{ Happy tweets } \\
\hline Third tertile (highest) & $-0.85(-1.48$ to -0.21$)$ & $0.92(0.82$ to 1.04$)$ & 0.94 (0.88 to 1.00$)$ & $0.90(0.76$ to 1.05$)$ \\
\hline Second tertile & $-0.65(-1.10$ to -0.20$)$ & 0.95 (0.86 to 1.04$)$ & 0.92 (0.86 to 0.98$)$ & $1.02(0.90$ to 1.15$)$ \\
\hline \multicolumn{5}{|l|}{ Physical activity tweets } \\
\hline Third tertile (highest) & $-0.57(-1.27$ to 0.12$)$ & $0.94(0.85$ to 1.04$)$ & $0.90(0.85$ to 0.96$)$ & 1.09 (0.87 to 1.37$)$ \\
\hline Second tertile & $-0.18(-0.83$ to 0.47$)$ & 1.00 (0.91 to 1.09$)$ & $0.92(0.87$ to 0.98$)$ & 1.09 (0.91 to 1.32$)$ \\
\hline \multicolumn{5}{|l|}{ Fast food tweets } \\
\hline Third tertile (highest) & $-0.37(-0.84$ to 0.11$)$ & 0.98 (0.90 to 1.07$)$ & $0.96(0.88$ to 1.04$)$ & $1.00(0.84$ to 1.19$)$ \\
\hline Second tertile & $-0.47(-1.04$ to 0.10$)$ & $0.99(0.89$ to 1.10$)$ & 0.95 (0.89 to 1.02$)$ & $1.00(0.83$ to 1.21$)$ \\
\hline \multicolumn{5}{|l|}{ Healthy food tweets } \\
\hline Third tertile (highest) & $-1.02(-1.75$ to -0.28$)$ & $0.88(0.79$ to 0.98$)$ & 0.99 (0.91 to 1.06$)$ & $1.00(0.83$ to 1.21$)$ \\
\hline Second tertile & $-0.73(-1.39$ to -0.07$)$ & 0.95 (0.86 to 1.04$)$ & $0.94(0.88$ to 1.00$)$ & $1.00(0.85$ to 1.16$)$ \\
\hline NHANES participants - $1^{\mathrm{c}, \mathrm{d}}$ & 15,897 & 15,897 & 15,412 & 15,473 \\
\hline NHANES participants $-2^{\mathrm{e}}$ & 15,774 & 15,774 & 15,291 & 15,353 \\
\hline
\end{tabular}

${ }^{\mathrm{a}}$ NHANES 2011-2016 among adults 20 years and older.

${ }^{\mathrm{b}}$ Adjusted regression models were run for each outcome. For dichotomous outcomes such as obesity and diabetes ( $0=$ no; $\left.1=y e s\right)$, Poisson models were utilized. For continuous variables like body mass index, linear regression was used. Models controlled for individual-level demographics including age, gender, race/ethnicity, annual household income, as well as zip code-level characteristics such as population density, percent white, median age, and median household income. Twitter-derived characteristics were categorized into tertiles, with the lowest tertile serving as the reference group. Analyses accounted for survey weights and complex survey design to produce nationally representative estimates.

${ }^{\mathrm{c}}$ NHANES: National Health and Nutrition Examination Survey.

${ }^{d}$ Number of NHANES participants included in models with zip code-level happy tweets or physical activity tweets as the predictor variable.

${ }^{\mathrm{e}}$ Number of NHANES participants included in models with zip code-level healthy food tweets or fast food tweets as the predictor variable. 
Table 5. Twitter-derived neighborhood characteristics and adult health outcomes in full cohort ${ }^{\mathrm{a}}$.

\begin{tabular}{|c|c|c|c|c|}
\hline $\begin{array}{l}\text { Zip code-level Twitter pre- } \\
\text { dictors and tertiles }\end{array}$ & BMI $\left(\mathrm{kg} / \mathrm{m}^{2}\right), \mathrm{b}(95 \% \mathrm{CI})^{\mathrm{b}}$ & $\begin{array}{l}\text { Obesity, prevalence ratio } \\
(95 \% \mathrm{CI})^{\mathrm{b}}\end{array}$ & $\begin{array}{l}\text { Hypertension, prevalence } \\
\text { ratio }(95 \% \mathrm{CI})^{\mathrm{b}}\end{array}$ & $\begin{array}{l}\text { Diabetes, prevalence ratio } \\
(95 \% \mathrm{CI})^{\mathrm{b}}\end{array}$ \\
\hline \multicolumn{5}{|l|}{ Happy tweets } \\
\hline Third tertile (highest) & $-0.79(-1.25$ to -0.33$)$ & $0.90(0.82$ to 0.98$)$ & 0.94 (0.89 to 0.99$)$ & 0.87 (0.77 to 0.99$)$ \\
\hline Second tertile & $-0.53(-0.81$ to -0.24$)$ & 0.93 (0.88 to 0.99$)$ & $0.94(0.89$ to 0.98$)$ & 0.99 (0.90 to 1.09$)$ \\
\hline \multicolumn{5}{|l|}{ Physical activity tweets } \\
\hline Third tertile (highest) & $-0.69(-1.19$ to -0.19$)$ & $0.89(0.82$ to 0.97$)$ & 0.91 (0.87 to 0.96$)$ & $1.04(0.87$ to 1.24$)$ \\
\hline Second tertile & -0.34 ( -0.80 to 0.12$)$ & 0.95 (0.89 to 1.02$)$ & $0.93(0.89$ to 0.97$)$ & $1.03(0.90$ to 1.18$)$ \\
\hline \multicolumn{5}{|l|}{ Fast food tweets } \\
\hline Third tertile (highest) & $-0.19(-0.60$ to 0.22$)$ & $1.00(0.93$ to 1.08$)$ & $0.95(0.89$ to 1.01$)$ & $1.05(0.91$ to 1.23$)$ \\
\hline Second tertile & $-0.26(-0.71$ to 0.18$)$ & $1.01(0.94$ to 1.10$)$ & $0.96(0.91$ to 1.02$)$ & $1.05(0.90$ to 1.23$)$ \\
\hline \multicolumn{5}{|l|}{ Healthy food tweets } \\
\hline Third tertile (highest) & $-1.02(-1.54$ to -0.51$)$ & $0.87(0.80$ to 0.94$)$ & $0.96(0.91$ to 1.01$)$ & $0.93(0.80$ to 1.09$)$ \\
\hline Second tertile & $-0.80(-1.26,-0.33)$ & $0.92(0.86,0.98)$ & $0.93(0.89,0.97)$ & $0.94(0.83,1.07)$ \\
\hline NHANES participants ${ }^{\mathrm{c}, \mathrm{d}}$ & 27,222 & 27,222 & 26,151 & 26,429 \\
\hline NHANES participants ${ }^{\mathrm{e}}$ & 26,814 & 26,814 & 25,752 & 26,029 \\
\hline
\end{tabular}

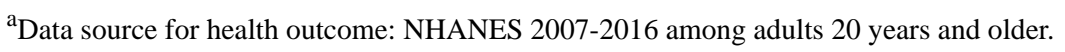

${ }^{\mathrm{b}}$ Adjusted regression models were run for each outcome separately. For dichotomous outcomes such as obesity and diabetes $(0=$ no; $1=y e s)$, Poisson models were utilized. For continuous variables like body mass index, linear regression was used. Models controlled for individual-level demographics including age, gender, race/ethnicity, annual household income, as well as zip code level characteristics including population density, percent of White, median age and median household income. Twitter-derived characteristics were categorized into tertiles, with the lowest tertile serving as the referent group. Analyses accounted for survey weights and complex survey design to produce nationally representative estimates.

${ }^{\mathrm{c}}$ NHANES: National Health and Nutrition Examination Survey.

${ }^{\mathrm{d}}$ Number of NHANES participants included in models with zip code-level happy tweets or physical activity tweets as the predictor variable.

${ }^{\mathrm{e}}$ Number of NHANES participants included in models with zip code-level healthy food tweets or fast food tweets as the predictor variable.

\section{Discussion}

This study is one of the first to investigate the relationship between Twitter-derived social neighborhood characteristics and individual cardiometabolic outcomes utilizing a nationally representative population. We found that healthy food was associated with lower mean BMI and lower prevalence of hypertension, and Twitter-derived physical activity was associated with a lower prevalence of hypertension. Associations between happy tweets and obesity and diabetes bordered statistical significance in the subcohort analyses (NHANES 2011-2016) but were statistically significant in the full cohort (NHANES 2007-2016). The associations between Twitter-derived characteristics and obesity were more evident in the full cohort than in the subcohort, possibly due to the larger sample size and higher statistical power.

Twitter-derived happiness was associated with lower mean BMI and lower prevalence of obesity and hypertension, suggesting the protective effect of positive emotion on obesity and hypertension. Results have also shown that neighborhoods with high and medium happiness tertiles have similar prevalence of obesity and hypertension, which indicates that the percent happiness in a neighborhood may not have any additional impact on cardiometabolic prevalence once it reaches a threshold. We included both continuous BMI and binary obesity as outcomes.
Our study results suggest that higher neighborhood happiness values shift BMI distributions lower. Individuals living in the third tertile have $0.85 \mathrm{~kg} / \mathrm{m}^{2}$ lower BMI than those living in the lowest tertile of neighborhood happiness. For obesity, this translates to an $8 \%$ lower relative risk. Although obesity is clinically important, the result of BMI provided insights for potential community interventions.

In our study, we focused on neighborhood-level happiness derived from Twitter, which is different from individual-level happiness. However, social networks spread happiness, and an individual's happiness is correlated to that of their neighbors, friends, and families [41]. The influence of affective state on outcomes via health behaviors could explain the link between happiness and a lower prevalence of health outcomes. Prior studies found negative emotions, including anger, depression, and anxiety, as well as stress, were associated with overeating, sedentary lifestyle, and physical inactivity [42-44]. Negative emotions and chronic stress may induce hemodynamic responses that lead to sustained elevation of blood pressure [45]. Although greater happiness is associated with lower cortisol and reduced plasma fibrinogen stress responses, indicating a lower risk for cardiovascular disease [46].

Associations between Twitter-derived physical activity mentions and lower hypertension suggest social learning of physical activity through Twitter may be effective at promoting the 
prevention of this condition. Health behaviors, including physical activity, occur in clusters rather than independently $[47,48]$. Information on physical activity and exercise behaviors may spread over the social network [49], and social network users are more likely to exercise if receiving repetitive messages on physical activity [50]. We also found Twitter-derived healthy food was associated with lower mean BMI and lower prevalence of obesity and hypertension. Social learning of healthy eating behaviors may help in shaping eating behaviors and consequently contribute to a lower prevalence of chronic health outcomes. Our results indicate the potential utility of Twitter as a platform to impact chronic disease prevention via behavioral changes.

Although not statistically significant, we observed associations between Twitter-derived social neighborhood characteristics and outcomes in unexpected directions. More fast food tweets were associated with lower mean BMI and lower prevalence of hypertension. Fast food consumption may be less affected by the local food environment but more affected by individual-level characteristics, including gender, socioeconomic status, and personal preferences [23,51-53]. Some fast-food tweets may come from advertisers rather than individual users. Healthy food tweets are generally sent by individual users, which may partially explain why healthy food tweets are significantly associated with certain community-level health outcomes, while fast food tweets are not. We also found a non-significant association between physical activity tweets and the prevalence of diabetes. We postulate that because diabetes is a complex condition affected by both genetics and environmental factors, the disease is unlikely to change swiftly or reflect the effect of the neighborhood environment.

It is important to note that this study is subject to several limitations. While Twitter does not record user demographics, Twitter users are generally younger [54], and there are more male Twitter users than females [55]. Twitter users are not a representative sample of the general population. Nonetheless, we argue that Twitter data, while imperfect, provides useful information about the social environment that corresponds with differentials in health outcomes [56]. In addition, we only collected geotagged tweets that had the latitude and longitude coordinates, representing a small fraction of all publicly available tweets. Thus, geotagged tweets may not fully capture the social environment for all Twitter users. Moreover, our keyword list approach to classification may not capture all tweets that fall within each topic or misidentify irrelevant tweets. However, we anticipate that misclassified tweets will comprise an insignificantly small portion of all tweets. Misclassification could also occur when assigning the sentiment score to a tweet due to the difficulty in recognizing and differentiating sarcastic expressions and humor. We performed validation for sentiment analysis comparing machine-labeled and manually labeled tweets and observed a high agreement between machine and manually labeled data [20].

Additionally, the study is observational and cross-sectional, which inhibits causal inference. We were unable to establish the temporality between Twitter-derived social neighborhood characteristics and cardiometabolic outcomes. To lessen discordance in the time frame and reduce the potential bias introduced from changing social environments, we implemented separate regression analyses for NHANES data from 2011 to 2016 and from 2007 to 2016. Results generally followed the same pattern for the two time periods, and we observed associations between Twitter-derived characteristics with obesity and hypertension.

We did not account for local resources that might influence cardiometabolic outcomes, for instance, the availability of grocery stores and local sources of healthy foods. However, we controlled for zip code-level characteristics, including percent non-Hispanic white, median age, population density, and median household income in the regression analyses.

Our study has several advantages. We utilize a publicly available big data source, allowing us to create neighborhood characteristics for small areas across the entire contiguous United States. This approach differs significantly from the majority of neighborhood studies that are restricted to local geography, given the time-consuming and expensive nature of gathering neighborhood data. Our study advances the use of social media in health research by constructing social neighborhood characteristics and applied these characteristics at individual-level quantitative analyses. Although researchers have been increasingly aware of the value of using social media data in health research, the majority of existing health studies are content analyses. We are not aware of any studies that used quantitative Twitter characteristics in individual-level outcome research. Additionally, leveraging individual data from NHANES allowed us to incorporate objective health assessments and extensive individual-level demographic information.

Our study investigated the relationships between Twitter-derived social neighborhood features and individual cardiometabolic outcomes in a nationally representative population. Our findings show Twitter as an emerging and cost-effective data source for public health that could be used to understand the potential influence of social context on important chronic health conditions. Researchers and public health practitioners may use Twitter as a public health surveillance tool to identify communities with greater risk of cardiometabolic outcomes. Practitioners could also utilize Twitter as a platform for health education and the social promotion of healthy behaviors aimed at reducing the burden for cardiometabolic outcomes.

\section{Acknowledgments}

The findings and conclusions in this paper are those of the authors. They do not necessarily represent the views of the Research Data Center, the National Center for Health Statistics, or the Centers for Disease Control and Prevention. The authors thank the staff of the RDC for their data support and technical assistance. 
This study was supported by the National Library of Medicine of the National Institutes of Health under award number [R01LM012849], the Big Data to Knowledge Initiative (BD2K) grant 5K01ES025433, and the NIH Commons Credit Pilot Program (grant number: CCREQ-2016-03-00003). QCN is the principal investigator for all three grants.

Data collection for NHANES was approved by the NCHS Research Ethics Review Board (ERB). Analysis of de-identified data from the survey is exempt from the federal regulations for the protection of human research participants. Analysis of restricted data through the NCHS Research Data Center is also approved by the NCHS ERB. The study was approved by the University of Maryland College Park (UMCP) IRB (IRB\#1304839-1).

\section{Authors' Contributions}

Conceptualization and methodology - QCN; data collection - SK and PD; formal analysis - DH; writing (original draft preparation) - DH, review and editing - DH, QCN, YH, NS, KMG, XH, RP; funding acquisition - QCN.

\section{Conflicts of Interest}

None declared.

\section{References}

1. Saelens BE, Handy SL. Built Environment Correlates of Walking. Medicine \& Science in Sports \& Exercise 2008;40(Supplement):S550-S566. [doi: 10.1249/mss.0b013e31817c67a4] [Medline: 18562973]

2. Sallis JF, Conway TL, Cain KL, Carlson JA, Frank LD, Kerr J, et al. Neighborhood built environment and socioeconomic status in relation to physical activity, sedentary behavior, and weight status of adolescents. Preventive Medicine 2018 May;110:47-54. [doi: 10.1016/j.ypmed.2018.02.009] [Medline: 29432790]

3. Alvarado SE. Neighborhood disadvantage and obesity across childhood and adolescence: Evidence from the NLSY children and young adults cohort (1986-2010). Social Science Research 2016 May;57:80-98. [doi: 10.1016/j.ssresearch.2016.01.008] [Medline: 26973033]

4. Barrientos-Gutierrez T, Moore K, Auchincloss A. Neighborhood Physical Environment and Changes in Body Mass Index: Results From the Multi-Ethnic Study of Atherosclerosis. American journal of epidemiology 2017;186(11):1237-1245. [doi: 10.1093/aje/kwx186] [Medline: 29206987]

5. Galea S, Ahern J, Rudenstine S, Wallace Z, Vlahov D. Urban built environment and depression: a multilevel analysis. Journal of Epidemiology \& Community Health 2005 Oct 01;59(10):822-827. [doi: 10.1136/jech.2005.033084] [Medline: 16166352]

6. Evans GW. The Built Environment and Mental Health. Journal of Urban Health: Bulletin of the New York Academy of Medicine 2003 Dec 01;80(4):536-555. [doi: 10.1093/jurban/jtg063] [Medline: 14709704]

7. Mujahid MS, Roux AVD, Shen M, Gowda D, Sanchez B, Shea S, et al. Relation between Neighborhood Environments and Obesity in the Multi-Ethnic Study of Atherosclerosis. American Journal of Epidemiology 2008 Mar 25;167(11):1349-1357. [doi: 10.1093/aje/kwn047] [Medline: 18367469]

8. Ding D, Sallis JF, Kerr J, Lee S, Rosenberg DE. Neighborhood Environment and Physical Activity Among Youth. American Journal of Preventive Medicine 2011 Oct;41(4):442-455. [doi: 10.1016/j.amepre.2011.06.036] [Medline: 21961474]

9. Cunningham-Myrie CA, Theall KP, Younger NO, Mabile EA, Tulloch-Reid MK, Francis DK, et al. Associations between neighborhood effects and physical activity, obesity, and diabetes: The Jamaica Health and Lifestyle Survey 2008. Journal of Clinical Epidemiology 2015 Sep;68(9):970-978. [doi: 10.1016/j.jclinepi.2014.08.004] [Medline: 25910912]

10. Corriere MD, Yao W, Xue QL, Cappola AR, Fried LP, Thorpe RJ, et al. The association of neighborhood characteristics with obesity and metabolic conditions in older women. J Nutr Health Aging 2015 Jan 8;18(9):792-798. [doi: 10.1007/s12603-014-0551-z] [Medline: 25389956]

11. Buys DR, Howard VJ, McClure LA, Buys KC, Sawyer P, Allman RM, et al. Association Between Neighborhood Disadvantage and Hypertension Prevalence, Awareness, Treatment, and Control in Older Adults: Results From the University of Alabama at Birmingham Study of Aging. Am J Public Health 2015 Jun;105(6):1181-1188. [doi: 10.2105/ajph.2014.302048] [Medline: 25322309]

12. Coulon SJ, Velasco-Gonzalez C, Scribner R, Park CL, Gomez R, Vargas A, et al. Racial differences in neighborhood disadvantage, inflammation and metabolic control in black and white pediatric type 1 diabetes patients. Pediatr Diabetes 2016 Jan 18;18(2):120-127. [doi: 10.1111/pedi.12361] [Medline: 26783014]

13. Burdette HL, Whitaker RC. A National Study of Neighborhood Safety, Outdoor Play, Television Viewing, and Obesity in Preschool Children. Pediatrics 2005 Sep 01;116(3):657-662. [doi: 10.1542/peds.2004-2443] [Medline: 16140705]

14. Egolf B, Lasker J, Wolf S, Potvin L. The Roseto effect: a 50-year comparison of mortality rates. Am J Public Health 1992 Aug;82(8):1089-1092. [doi: 10.2105/ajph.82.8.1089] [Medline: 1636828]

15. Oswald A, Powdthavee N. Obesity, unhappiness, and the challenge of affluence: Theory and evidence. IZA Discussion Paper No. 2717 2007. [doi: 10.1093/acprof:oso/9780199216628.001.0001] 
16. Bray I, Gunnell D. Suicide rates, life satisfaction and happiness as markers for population mental health. Soc Psychiat Epidemiol 2006 Mar 25;41(5):333-337. [doi: 10.1007/s00127-006-0049-z] [Medline: 16565916]

17. Blanchflower DG, Oswald AJ. Hypertension and happiness across nations. Journal of Health Economics 2008 Mar;27(2):218-233. [doi: 10.1016/j.jhealeco.2007.06.002] [Medline: 18199513]

18. Kawachi I, Subramanian S, Kim D. Social capital and health. New York: Springer; 2008:1-26.

19. Hamano T, Fujisawa Y, Yamasaki M, Ito K, Nabika T, Shiwaku K. Contributions of social context to blood pressure: findings from a multilevel analysis of social capital and systolic blood pressure. American journal of hypertension 2011;24(6):643-646. [doi: 10.1038/ajh.2011.37] [Medline: 21415843]

20. Nguyen QC, Li D, Meng H, Kath S, Nsoesie E, Li F, et al. Building a National Neighborhood Dataset From Geotagged Twitter Data for Indicators of Happiness, Diet, and Physical Activity. JMIR Public Health Surveill 2016 Oct 17;2(2):e158. [doi: 10.2196/publichealth.5869] [Medline: 27751984]

21. Abbar S, Mejova Y, Weber I. You Tweet What You Eat: Studying Food Consumption Through Twitter. 2015 Presented at: Proceedings of the 33rd Annual ACM Conference on Human Factors in Computing Systems; 2015; Seoul p. $3197-3206$. [doi: $10.1145 / 2702123.2702153]$

22. Mitchell L, Frank MR, Harris KD, Dodds PS, Danforth CM. The Geography of Happiness: Connecting Twitter Sentiment and Expression, Demographics, and Objective Characteristics of Place. PLoS ONE 2013 May 29;8(5):e64417. [doi: 10.1371/journal.pone.0064417] [Medline: 23734200]

23. Widener MJ, Li W. Using geolocated Twitter data to monitor the prevalence of healthy and unhealthy food references across the US. Applied Geography 2014 Oct;54:189-197. [doi: 10.1016/j.apgeog.2014.07.017]

24. Bandura A, Walters RH. Social learning theory (Vol. 1). Prentice-Hall: Englewood Cliffs, NJ; 1977.

25. The Facts About High Blood Pressure. URL: http://www.heart.org/HEARTORG/Conditions/HighBloodPressure/ GettheFactsAboutHighBloodPressure/The-Facts-About-High-Blood-Pressure_UCM_002050_Article.jsp\#.Wt4bJ24vypo [accessed 2018-04-22]

26. Brownson RC, Hoehner CM, Day K, Forsyth A, Sallis JF. Measuring the Built Environment for Physical Activity. American Journal of Preventive Medicine 2009 Apr;36(4):S99-S123.e12. [doi: 10.1016/j.amepre.2009.01.005]

27. Roemmich JN, Epstein LH, Raja S, Yin L, Robinson J, Winiewicz D. Association of access to parks and recreational facilities with the physical activity of young children. Preventive Medicine 2006 Dec;43(6):437-441. [doi: 10.1016/j.ypmed.2006.07.007] [Medline: 16928396]

28. Van RG, Drake F. Python 3 Reference Manual. Scotts Valley, CA: CreateSpace; 2009.

29. McCallum A. MALLET: A Machine Learning for Language Toolkit. Amherst, MA: http://mallet.cs.umass.edu; 2002.

30. Christiansen KM, Qureshi F, Schaible A, Park S, Gittelsohn J. Environmental Factors That Impact the Eating Behaviors of Low-income African American Adolescents in Baltimore City. Journal of Nutrition Education and Behavior 2013 Nov;45(6):652-660. [doi: 10.1016/j.jneb.2013.05.009] [Medline: 23916684]

31. Sanders NJ. Sanders-Twitter Sentiment Corpus.: Sanders Analytics LLC URL: http://www.sananalytics.com/lab/ twitter-sentiment/ [accessed 2018-11-30]

32. Kaggle UMICH SI650 - Sentiment Classification. 2011. URL: https://www.kaggle.com/c/si650winter11 [accessed 2018-11-30]

33. Nguyen QC, Kath S, Meng H, Li D, Smith KR, VanDerslice JA, et al. Leveraging geotagged Twitter data to examine neighborhood happiness, diet, and physical activity. Applied Geography 2016 Aug;73:77-88. [doi:

10.1016/j.apgeog.2016.06.003] [Medline: 28533568]

34. McIver DJ, Hawkins JB, Chunara R, Chatterjee AK, Bhandari A, Fitzgerald TP, et al. Characterizing Sleep Issues Using Twitter. J Med Internet Res 2015 Jun 08;17(6):e140. [doi: 10.2196/jmir.4476] [Medline: 26054530]

35. Compendium of Physical Activities. 2011. URL: https://sites.google.com/site/compendiumofphysicalactivities/home [accessed 2018-11-30]

36. Zhang N, Campo S, Janz KF, Eckler P, Yang J, Snetselaar LG, et al. Electronic Word of Mouth on Twitter About Physical Activity in the United States: Exploratory Infodemiology Study. J Med Internet Res 2013 Nov 20;15(11):e261. [doi: 10.2196/jmir.2870] [Medline: 24257325]

37. Ainsworth B, Haskell W, Herrmann S. 2011 Compendium of Physical Activities: A Second Update of Codes and MET Values. Med Sci Sports Exerc 2011;43(8):1575-1581. [doi: 10.1249/mss.0b013e31821ece12] [Medline: 21681120]

38. Barros AJ, Hirakata VN. Alternatives for logistic regression in cross-sectional studies: an empirical comparison of models that directly estimate the prevalence ratio. BMC Med Res Methodol 2003 Oct 20;3(1). [doi: 10.1186/1471-2288-3-21] [Medline: 14567763]

39. National Health and Nutrition Examination Survey: Estimation Procedures 2007-2010. URL: https://www.cdc.gov/nchs/ data/series/sr 02/sr02 159.pdf [accessed 2018-11-30]

40. National Health and Nutrition Examination Survey: Estimation Procedures 2011-2014. URL: https://www.cdc.gov/nchs/ data/series/sr 02/sr02 177.pdf [accessed 2018-11-30]

41. Fowler JH, Christakis NA. Dynamic spread of happiness in a large social network: longitudinal analysis over 20 years in the Framingham Heart Study. BMJ 2008 Dec 04;337:a2338. [doi: 10.1136/bmj.a2338] [Medline: 19056788] 
42. Anton SD, Miller PM. Do Negative Emotions Predict Alcohol Consumption, Saturated Fat Intake, and Physical Activity in Older Adults? Behav Modif 2016 Jul 26;29(4):677-688. [doi: 10.1177/0145445503261164] [Medline: 15911688]

43. Carney RM, Freedland KE, Rich MW, Jaffe AS. Depression as a risk factor for cardiac events in established coronary heart disease: A review of possible mechanisms. Ann Behav Med 1995 Jun;17(2):142-149. [doi: 10.1007/bf02895063] [Medline: $\underline{18425665]}$

44. Rozanski A, Blumenthal JA, Kaplan J. Impact of Psychological Factors on the Pathogenesis of Cardiovascular Disease and Implications for Therapy. Circulation 1999 Apr 27;99(16):2192-2217. [doi: 10.1161/01.cir.99.16.2192] [Medline: 10217662]

45. Sparrenberger F, Cichelero FT, Ascoli AM, Fonseca FP, Weiss G, Berwanger O, et al. Does psychosocial stress cause hypertension? A systematic review of observational studies. J Hum Hypertens 2008 Jul 10;23(1):12-19. [doi: 10.1038/jhh.2008.74] [Medline: 18615099]

46. Steptoe A, Wardle J, Marmot M. Positive affect and health-related neuroendocrine, cardiovascular, and inflammatory processes. Proceedings of the National Academy of Sciences 2005 Apr 19;102(18):6508-6512. [doi: 10.1073/pnas.0409174102]

47. Vickers RR, Conway TL, Hervig LK. Demonstration of replicable dimensions of health behaviors. Preventive Medicine 1990 Jul;19(4):377-401. [doi: 10.1016/0091-7435(90)90037-k] [Medline: 2399221]

48. Langlie J. Interrelationships among preventive health behaviors: a test of competing hypotheses. Public Health Reports 1979;94(3):216-225. [Medline: 451130]

49. Pagoto S, Schneider K, Oleski J, Smith B, Bauman M. The adoption and spread of a core-strengthening exercise through an online social network. Journal of physical activity \& health 2014;11(3):648-653. [doi: 10.1123/jpah.2012-0040] [Medline: 23416874]

50. Centola D. The Spread of Behavior in an Online Social Network Experiment. Science 2010 Sep 02;329(5996):1194-1197. [doi: $10.1126 /$ science.1185231]

51. Jeffery RW, Baxter J, McGuire M, Linde J. Are fast food restaurants an environmental risk factor for obesity? Int J Behav Nutr Phys Act 2006 Jan 25;3:2 [FREE Full text] [doi: 10.1186/1479-5868-3-2] [Medline: 16436207]

52. Driskell JA, Meckna BR, Scales NE. Differences exist in the eating habits of university men and women at fast-food restaurants. Nutrition Research 2006 Oct;26(10):524-530. [doi: 10.1016/j.nutres.2006.09.003]

53. Glanz K, Basil M, Maibach E, Goldberg J, Snyder D. Why Americans Eat What They Do : Taste, Nutrition, Cost, Convenience, and Weight Control Concerns as Influences on Food Consumption. Journal of the American Dietetic Association 1998 Oct;98(10):1118-1126. [doi: 10.1016/s0002-8223(98)00260-0] [Medline: 9787717]

54. Duggan M. Mobile Messaging and Social Media 2015. URL: http://www.pewinternet.org/2015/08/19/ mobile-messaging-and-social-media-2015/ [accessed 2018-04-22]

55. Understanding the Demographics of Twitter Users. URL: https://www.aaai.org/ocs/index.php/ICWSM/ICWSM11/paper/ view/2816/3234 [accessed 2020-03-20]

56. Ball K, Jeffery RW, Abbott G, McNaughton SA, Crawford D. Is healthy behavior contagious: associations of social norms with physical activity and healthy eating. Int J Behav Nutr Phys Act 2010;7(1):86. [doi: 10.1186/1479-5868-7-86] [Medline: 21138550]

\section{Abbreviations}

API: application programming interface

MEC: Mobile Examination Center

NCHS: National Center for Health Statistics

NHANES: National Health and Nutrition Examination Survey

RDC: Restricted Data Center

Edited by T Rashid Soron; submitted 29.01.20; peer-reviewed by MG Kim, M Alvarez de Mon, J Colditz; comments to author 10.03.20;
revised version received 26.04.20; accepted 27.05.20; published 18.08 .20
Please cite as:
Huang D, Huang Y, Khanna S, Dwivedi P, Slopen N, Green KM, He X, Puett R, Nguyen Q
Twitter-Derived Social Neighborhood Characteristics and Individual-Level Cardiometabolic Outcomes: Cross-Sectional Study in a
Nationally Representative Sample
JMIR Public Health Surveill 2020;6(3):e17969
URL: $\underline{\text { http://publichealth.jmir.org/2020/3/e17969/ }}$
doi: $\underline{10.2196 / 17969}$
PMID: $\underline{32808935}$


CDina Huang, Yuru Huang, Sahil Khanna, Pallavi Dwivedi, Natalie Slopen, Kerry M Green, Xin He, Robin Puett, Quynh Nguyen. Originally published in JMIR Public Health and Surveillance (http://publichealth.jmir.org), 18.08.2020. This is an open-access article distributed under the terms of the Creative Commons Attribution License (https://creativecommons.org/licenses/by/4.0/), which permits unrestricted use, distribution, and reproduction in any medium, provided the original work, first published in JMIR Public Health and Surveillance, is properly cited. The complete bibliographic information, a link to the original publication on http://publichealth.jmir.org, as well as this copyright and license information must be included. 